\title{
Visual decisions: Geovisualisation techniques within retail decision support
}

Received (in revised form): 7th February, 2005

\section{Tony Hernandez}

is the Associate Eaton Chair in Retailing at the Centre for the Study of Commercial Activity, and Associate Professor in the Department of Geography, Ryerson University, Toronto. He works on a number of public and private sector-funded research projects in the area of business geomatics, specialising in location analysis and decision support. Dr Hernandez teaches courses on geodemographics, market segmentation and retail location analysis at the undergraduate and graduate level.

\begin{abstract}
Geovisualisation refers to an established area of research that provides the theory, methods and tools for visual exploration, analysis, synthesis and presentation of geospatial data. The aim of geovisualisation is to facilitate the knowledge-creation process by providing techniques and technologies that allow decision makers to interact visually with data by displaying the data in ways that promote visual-cognitive learning. The applications of geovisualisation within the retail industry are many and varied, for example, rendering complex retail demand surfaces (eg probabilistic trade areas) over time around a network of stores, sequencing changes in retail metrics (eg sales per square foot, profitability by store) and monitoring change in commercial structure (eg retail vacancy/turnover, new format retail development). To date, geographical information systems (GIS) technology in use within major retail organisations has relatively limited 'visual' capabilities, and finds dealing with temporal dimensions (ie the dimension upon which retail change occurs) particularly difficult. The increasing availability of time-series data within retail organisations provides many challenges and opportunities to the retail location analyst. It allows decision makers to move away from simply generating static 'snapshots' to undertaking 'dynamic' visualisation of change, for example, 3D animation of data sets over a number of time periods. This paper details the development of a prototype geovisualisation system that has been designed to enable geospatial visualisation of retail change. A number of potential application areas are examined. The paper concludes by highlighting the challenges that are faced in developing spatial-temporal visualisation systems and the potential to integrate such technology within retail decision support systems.
\end{abstract}

Tony Hernandez Centre for the Study of Commercial Activity (CSCA), Ryerson University, 350 Victoria Street, Toronto, M5B 2K3, Canada.

Tel: +1 4169795000 Ext. 7200 ;

Fax: +1 4169795378 ; e-mail: thernand@research. ryerson.ca

\section{GEOVISUALISATION AND RETAIL DECISION MAKING}

Retail and service firms represent a major user group of geographic information systems (GIS), with applications including trade area analysis, customer profiling and property portfolio mapping. ${ }^{1}$ The vast majority of GIS use within the retail sector has traditionally focused on rudimentary inventory-type mapping and analysis, with a relatively small number of retail organisations pursuing more complex modelling applications. Location-based decisions have often been supported by a range of static maps, for example, a 
map of the five top customer profile neighbourhoods within a given trade area, market share and penetration maps and simple customer spotting maps. Change in market conditions, such as sales change for a network of stores, the entry of new competitors or change in the demand profile (spending habits, demographic shifts) of the customer base over time have been represented as temporal snapshots (eg separate maps for each year between $\mathrm{t}_{1} \ldots \mathrm{t}_{\mathrm{n}}$ ) or relative changes (eg percentage change between time period $\mathrm{t}_{1}$ and $\mathrm{t}_{\mathrm{n}}$ ). To date, managing, analysing and visualising one of the most fundamental elements of the retail environment - change over time and space - has been difficult within a conventional GIS framework. ${ }^{2}$ The lack of such decision support capability (temporal, spatial and visual) presents a significant hurdle, and conversely, source of potential competitive advantage, to retail organisations. As more retailers collect and have access (often as a bi-product of day-to-day operations) to complex spatial-temporal data (ie highly detailed customer loyalty data, retail store sales and merchandise data, demographic projection data, retail spend data) the potential to develop systems that enable analysts and decision makers to manage, explore, analyse, synthesise and present such data are likely to increase. The 'data-rich' yet 'information-poor' decision environment outlined by Hernandez ${ }^{3}$ still exists within many retail organisations. This paper presents findings from research into the development and application of geovisualisation decision support systems for use within retail decision making. The aim of the paper is to provide insight into the potential application and functionality of geovisualisation systems and the myriad challenges that are faced in their development and deployment.

Geographical visualisation (geovisualisation) of spatially-related data has become an established area of geographic information science research. The primary aim of geovisualisation is to turn large heterogeneous data into information (interpreted data) and, subsequently, into knowledge (understanding derived from information). As MacEachren and $\mathrm{Kraak}^{4}$ define, 'geovisualization integrates approaches from visualization in scientific computing, cartography, image analysis, information visualisation, exploratory data analysis and geographic information systems to provide theory, methods and tools for visual exploration, analysis, synthesis and presentation of geospatial data'. ${ }^{5-10}$ Figure 1 provides a functional framework for geovisualisation systems, defined along three axes: (i) the nature of the tasks performed; (ii) the type of users; and, (iii) the level of interaction with the data. The four primary functions of geovisualisation can be placed along the central diagonal of the geovisualisation use space, ie to explore, analyse, synthesise and present spatial data. ${ }^{11,12}$ From an explicitly visual paradigm, geovisual techniques and technologies are geared towards exploiting the complex cognitive and visual processing abilities of the human brain to recognise, order and interpret data. They are based on the premise that humans learn more effectively and efficiently within a visual as opposed to textual or numerical setting. ${ }^{13-15}$ This serves to transform decision support from static unidimensional space into a dynamic environment that enables users to dynamically (interactively) explore multidimensional space. It should be noted, however, that such a visual paradigm is also subject to the dangers 


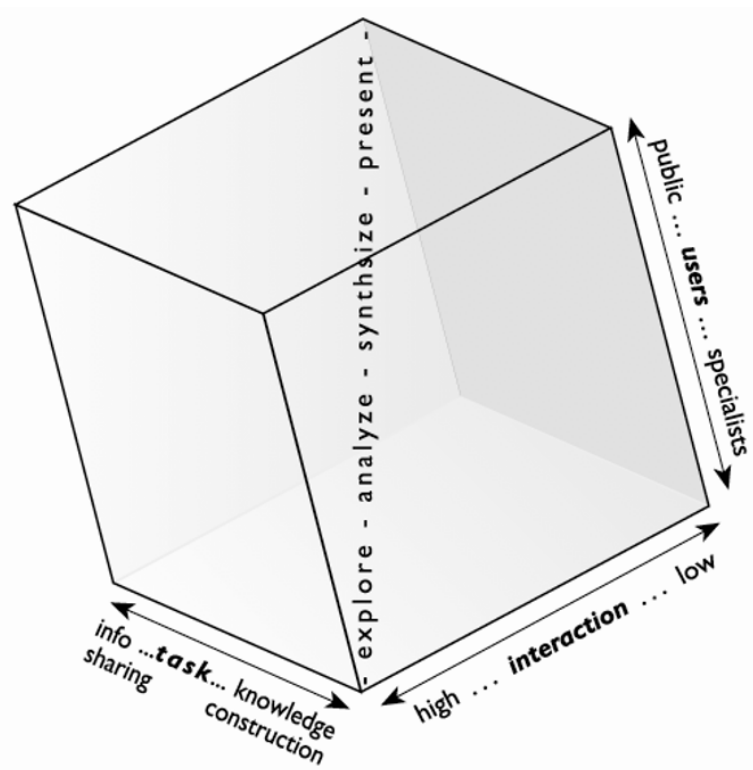

Figure 1 Functions of geovisualisation Source: MacEachren et. al. (2003)

of distorting information through ineffective 'technology-push' visualisation.

\section{DEVELOPING GEOVISUALISATION DECISION SUPPORT SYSTEMS}

This section outlines the development of a prototype geovisualisation system for use within retail applications - the 4D Geovisualizer. Three-dimensional mapping and spatial-temporal animation techniques are examined to illustrate the type of functionality that geovisualisation systems can provide. Typically, spatial data have been depicted from an abstract $2 \mathrm{D}$ plan view, such as a choropleth map that is viewed from directly overhead and represents data values through colour or shading. Vision has been the primary means of acquiring spatial knowledge. ${ }^{16}$ Three-dimensional mapping potentially assists in identifying spatial trends, intensity and variation in data. There have been a number of studies that have focused on the utilisation and potential for 3D mapping. ${ }^{17-19}$ Often data sets are just too complex for representation in two or even three dimensions. By animating the display across user-defined independent variables, users can easily observe trends in extremely complex data sets. With the addition of temporal-animation (4D), the user has the ability to discover trends, patterns and anomalies in data. The term 'dynamic representation' refers to displays that change continuously, either with or without user control. Dynamic data representation has changed the way users obtain and interact with information across the full range of display technologies. ${ }^{20,21}$ One form of dynamic representation is the animated map, in which a display changes continuously, either with or without the user having control over that change. ${ }^{22,23}$ An argument for utilising animation is that it is natural for depicting temporal data. ${ }^{24-26}$ This is because changes in real-world time can be reflected by changes in display time. In addition to enabling animated maps, dynamic representations 
also permit users to explore geospatial data by interacting with mapped displays, a process sometimes referred to as direct manipulation. ${ }^{27}$ Studies to date on the merits of 3D mapping and temporal-animation vary significantly, some advocate such techniques, while others highlight their limitations, ${ }^{28-32}$ such as, the difficulty of tracking changing data values on screen while simultaneously trying to interpret the size, shape and colour of visual objects.

The 4D Geovisualizer system outlined in this paper has been developed within the Iris Explorer Visualization programing environment. This modular object-oriented type programing environment allows system developers to create a series of customised objects and to link these within the Iris Explorer programming interface. In essence, the visualisation tasks are conceptualised as a series of objects, and these objects communicate with one another via information flows, with pipelines between the objects being able to accommodate a range of data and parameter types, for example, objects to read in spatial data, to create $3 \mathrm{D}$ symbols, to create animations of data, etc. The final flow of data is typically into a render object or some form of data output through which a variety of information can be displayed (including maps, tables, graphs, multimedia files, etc). The benefit of modular design is in the ability to be able to modify existing modules in order to create customised packaged applications, for example, providing retail analyst users with access to the entire set of modules and restricting retail executives to a small subset of 'black-box' display modules.

As the system prototype described in this paper has been geared towards the business GIS user, the development of the system within the Windows operating environment was seen as a key element in terms of testing and evaluating the system among, primarily, analysts and decision makers within retail organisations. The majority of retail GIS users work on the PC platform. To date, the majority of research within geovisualisation has taken place within a scientific workstation environment. This has traditionally been seen as a major hurdle to the diffusion of geovisualisation systems for use within business, due to the relatively high cost of such specialised scientific workstations.

\section{Geovisualisation techniques}

This section outlines some of the key components of the 4D Geovisualizer system that have been developed to date, demonstrating the type of functionality that can be incorporated within geovisualisation applications. An overview of the system design is provided, and a number of the core issues/challenges that are faced in developing geovisualisation systems are examined. First, however, it should be noted that the data hurdles encountered when using GIS (eg it is not unusual to spend 80 to 90 per cent of GIS research time managing data, and only 10 to 20 per cent actually undertaking analysis) increase exponentially when dealing with geovisualisation systems. A significant barrier to the development of visualisation has been the difficulty of working between standard GIS formats and the data structures required by the leading visualisation software packages (eg Iris Explorer, AVS, IBM Data Explorer, etc). The data structures employed within these software packages do not support the easy translation from existing GIS packages. As a result, a number of customised data translators were created to allow users to work directly with standard GIS formats. The translators create a number of files that store the 
topology of the map objects in a customised spatial-temporal structure.

\section{Three-dimensional mapping}

Three-dimensional mapping allows the user to visualise data values effectively by scaling the $\mathrm{Z}$ axis of each object (eg a polygon object representing a postal area, or point object representing a store). For example, a map of average income at the enumeration level could be converted into $3 \mathrm{D}$ by scaling the $\mathrm{Z}$ axis relative to the value in each census area (ie creating a prism type map - this static function is available in many GIS packages). In order to convert 2D GIS maps into 3D maps a number of geometric objects need to be created. As is common practice in computer graphics programming, 2D polygons (eg GIS mapped census areas) need to be triangulated, with each polygon divided into the minimum set of triangles that define the area - to create a base triangulated map layer. O'Rourke ${ }^{33}$ provides an excellent primer to polygon triangulation algorithms.

It should be noted that the creation of 3D objects creates significant computational overheads, specifically, processing and graphics memory loads in part explaining why geovisualisation has traditionally taken place on high-end scientific workstations. For example, a basic map of Forward Sortation Area (FSA) postal boundaries for all of Canada (approximately 1,600 polygons) will create somewhere in the order of two to three million triangles (due to Canada's complex coastal boundaries). For most geovisualisation applications it is appropriate to generalise the polygons, that is, simplify the geometric shapes, prior to translating the files into 3D objects. Numerous algorithms are available, and a number of these are available within standard GIS packages.
The challenge with generalisation is in minimising geometric complexity, while maintaining the integrity of the geographical shape and the topology of the entire data set (eg simplifying boundaries should not interfere with a user's abilities to recognise the area). An approach that has worked effectively is to use a detailed geometric shape file as a cosmetic map layer and generalise the file that will be used to create the 3D objects. In this way, the user is able to view the detail of original boundaries as a background map with the simplified 3D objects placed above. The GeoPoly module allows users to create $3 \mathrm{D}$ prism maps seamlessly.

Three-dimensional point representation allows the user to choose a number of 3D shapes to display point-based data, and can be used as an alternative or viewed simultaneously with $3 \mathrm{D}$ prism maps. These points could be centroids for the polygons (ie using points to display areal data) or actual locations (eg a geocoded record for a large format retailer or shopping mall). A number of 3D point shapes have been implemented in the GeoPoint module, including pyramids, cubes, octas and spheres $(2 \mathrm{D}$ versions of these shapes are also available, ie simple point, circle, triangle, square and diamond). In the case of spheres, the user can set the level of geometric complexity of the sphere, which can range from very smooth complex spheres to highly orthogonal shapes. In addition, the GeoBar module allows users to create $3 \mathrm{D}$ shapes by selecting variables to represent the height, width, colour and complexity of the 3D object. For example, a set of store locations could be mapped with the height of the 3D cylinder representing total sales, the width of the cylinder indicating sales per square foot, the colour of the cylinder showing the percentage of customers over 65 and the geometric complexity of 


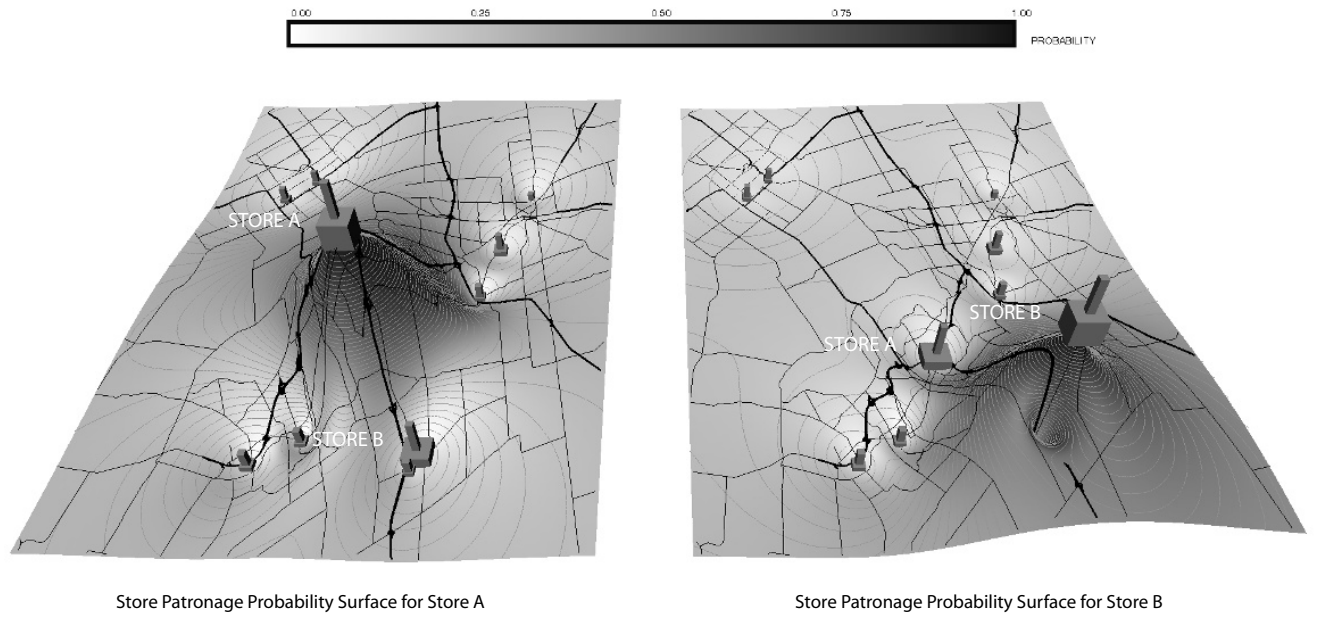

Figure 2 A comparison of probabilistic trade areas Note: The surface and associated contour lines represent the probability of store patronage at 'Store A' and 'Store B' respectively (see legend)

Store locations are represented as proportional 3D cubes, to indicate own and competitor stores ranging from 1,700 to 10,000 square feet

Source: The diagram was produced using CSCA GeoVisualizer software, built on the NAG Iris Explorer development platform (www.businessgeomatics.com, www.nag.co.uk)

the cylinder the store's competitive index.

The user can simultaneously view polygon and point data, with the time period of the data synchronised to allow the animation of temporal sequences. For example, 3D polygons may represent the total population by region, whereas 3D octas (diamond shapes) may show the population per square kilometre, therefore permitting the user simultaneously to view changes in both variables. In order to work within this multidimensional space, the user can either create separate windows or layer objects within the same window. The various objects can be offset on the $\mathrm{Z}$ axis, this provides the user with a way of layering data objects on top of each other. In addition, the transparency of any of the objects can be changed - for example, the user may want to focus on $3 \mathrm{D}$ point data, but also see the basic outline of the 3D polygon data.

An example of $3 \mathrm{D}$ visualisation is provided in Figure 2 that depicts a basic comparative $3 \mathrm{D}$ view of the retail trade areas for two stores operating within the same market. The size of the $3 \mathrm{D}$ cubes represents the size of the retail store in square feet, positioned on a probabilistic trade area surface. The probabilistic trade area surfaces were generated using a very basic Huff-type spatial interaction model. Figure 2 shows the different shape and size of the trade area for 'Store A' and 'Store B' with the contour lines also highlighting variation in the surface. It is interesting to note the hollow in the surface next to 'Store B' which captures the impact of a nearby competitor (with the likelihood of customers visiting 'Store B' decreasing sharply around the competitor store). This type of visualisation allows a retail analyst quickly to compare the trading environment of two or more stores (or two or more trade area models for the same store).

\section{Spatial-temporal animation}

A standard set of video recorder type buttons to navigate the temporal dimension of the data are provided in the 
GeoWorkbench module, eg forward and backward display of the temporal sequence, controls (widgets) to change the start and end time periods, the step between time periods and time delay between periods. In order to provide the user with a smooth animation, however, it is often necessary to interpolate a number of virtual values between actual values. For example, a user wants to display a map of store sales for 30 outlets, covering ten years of annual sales data. If the user created an annual sequence of these data, there would be ten time snapshots. It is likely that such a sequence would not appear particularly smooth, ie the data would change abruptly ten times, an alternative approach is to create an additional sequence of interim virtual snapshots between each actual time snapshot - eg creating nine additional sequences between each time period that would result in the map window updating 100 times. The ability to produce animations of data sequences and produce digital movie files is particularly important in terms of the sharing of information between different departments or individuals within a given company. For example, an analyst may produce an animated data movie showing the growth of their store network and the changing level of customer penetration measured on a monthly basis over a three-year time frame for a capital investment board meeting.

The need for dynamic legends when dealing with temporal data has been the focus of a number of studies. ${ }^{34-36}$ In the case of the static map, only a single legend is required for the single array of data values. When visualising a series of time periods (snapshots), multiple arrays of data values are being displayed; therefore a legend with a minimum and maximum value for time period $t_{1}$ may not cover the range of data in time period $t_{2}$ to $t_{n}$. There are two approaches that can be adopted to address this problem. First, the legend is updated for every new time period (dynamic) - this can be very difficult for the end-user to interpret as the objects on the map are changing shape and colour while the legend is also changing, which clearly creates difficulties for the user (particularly if the data are only displayed for a short period of time). Simply, the user has little time to make sense of the map. The second approach is to take the minimum and maximum values of the data values across all of the data arrays and keep the legend static (fixed). In this case, if the data range is large, or there is sporadic or dramatic change in the data values, the fixed legend may not provide useful information across a number of the time periods. An issue with displaying a number of variables simultaneously is that the user requires a legend or some other means of assessing the range and intensity of the values being displayed, for example, simultaneously displaying four variables require four associated legends - this again can become overwhelming for the user.

\section{GIS functionality}

In addition to providing the ability to create 3D maps and navigate sequences of data over time, a set of basic GIS functions are also required in order to allow the user to interact dynamically with the system. It should be noted that the purpose here is not to recreate GIS software - the aim of geovisualisation is concerned with visually exploring data for relationships and not solely providing inventory type functionality. Additional functions include basic layering options which allow maps layers to be switched on and off, the ability to manipulate data legend, class intervals, text, the colour and size of cosmetic map objects, etc. Basic click-and-display query functionality 
allows the user to click the mouse over any data object on the map and to display basic information, eg a simple descriptor of the polygon, the data values for the variable chosen. The GeoDisplay modules also have a standard set of tools for zooming in and out, panning, fly-to navigation, changing the background colour, changing axis manipulation, saving or printing the current render window as a standard image file, etc.

\section{GEOVISUALISATION APPLICATIONS}

A number of example applications have been created using a variety of publicly available temporal data sets. In addition, a range of company specific data sets have been made available to the research team (eg sales by store data, sales by category, store square footage, customer spend, etc). Examples of geovisualisation applications are available on the research project's website - a series of movie files can be downloaded from this website demonstrating the 4D animation sequencing. ${ }^{37}$ These techniques have been used to explore visually the growth of population across the USA from the early 1800 s to the present day — with the addition of population projection data, the user is able to continue visualising into the future. For example, proportional spheres can be used to indicate population per square kilometre and the height of the $3 \mathrm{D}$ polygons the total population by state. Geovisualisation has been applied to the Small Area Retail Trade Statistics (SARTRE) data set released by Statistics Canada. This national coverage data, disaggregated to the FSA level allows retailers to analyse the growth/decline of major retail sectors in terms of number of stores and retail sales (as defined by 70 standard industrial codes). A customised interface allows users to explore Canadian sales data at the national level from 1989 to 2001. For example, allowing the basic mapping of two variables, total retail sales (proportional 3D cubes) and number of retail stores (height of the 3D cylinders) - retailers can superimpose their own store network data onto the SARTRE data and assess if they have been developing stores in the fastest growing areas.

Geovisualisation has also been applied to the CSCA's retail database for the Greater Toronto Area (GTA). This retail application has provided a simple data visualisation tool to explore the changing retail structure of the GTA, for example, showing the growth and decline of ethnic retail, the widespread development of power centres, fluctuations in retail vacancy rates by shopping centre and along retail strips. Animation and 4D techniques can be applied also at the micro-level, for example a shopping centre manager wants to analyse the fluctuation of tenants' retail sales on a monthly basis over a five-year period pedestrian flow data could also be incorporated allowing the user to explore the link between sales and pedestrian traffic. Alternatively, a retail organisation may want to visualise the performance of their property portfolio by analysing differences between the various retail banners that they operate. The range of applications that can be developed are primarily limited by the availability of consistent high quality spatial-temporal data sets.

\section{THE CHALLENGE OF VISUAL DECISION MAKING}

This paper has outlined the potential for further development of geovisualisation techniques for use within the decision support activities of major retail and service firms. The benefits of the geovisualisation approach have been outlined, including for example, the 
ability to explore spatial-temporal data dynamically, the multidimensional display of complex data sets, the sequencing and animation of spatial-temporal data to visually uncover trends and identify anomalies. There are many potential application areas for geovisualisation systems within the retail and service industries; with such systems providing the ability to visually track the demand and supply of goods and services across a network of stores, and essentially provide the techniques and technology to geovisualise change over time.

There are, however, a number of significant barriers that continue to impede the development of geovisualisation systems, these include a range of technical, cognitive and economic factors; for example, the need for higher-end computing capacity and expertise, decision-makers' cognitive abilities to work in multidimensional space and comprehend sequences of data, and the cost-benefit rationale for investing in such decision support infrastructure, ie how much does the geovisual approach add to the decision outcome, and at what cost?

Arguably, the GIS industry has been slow to adopt 3D and spatial-temporal (4D) functionality, the majority of which produces static scenes (eg animated fly-through of static data, or non-interactive animation of time). As the technical barriers become less significant, the future development of geovisualisation and, specifically, the speed and diffusion of system development within the mainstream will be largely a function of the level of demand for such systems and the ability to provide generic systems (in terms of data portability and functionality). This results in somewhat of a 'chicken-and-an-egg' situation: without available geovisualisation systems users are not aware of potential benefits; without user demand it is difficult to justify new product development. This is particularly the case within the retail industry - that has been traditionally slow to adopt new information technology. It should be noted that a number of major retailers in North America and Europe made substantial investments in GIS infrastructure through the 1980s, with these early adopters investing heavily in scientific workstation GIS. The desktop GIS revolution of the 1990s, for the most part, limited the high-end GIS market. To date, retailers are either unaware of the potential of geovisualisation or unconvinced of the 'decision-making' benefits of investing in such technology.

This paper has outlined the development of a prototype system with a number of 'core' geovisualisation functions - sequencing, animation, temporal interpolation, 3D mapping, etc. Further prototype testing and development is currently underway with a number of retailers to examine the integration of such technology within decision support activities and to assess the 'value-add' provided by such systems. The findings will provide further evidence as to the viability of developing systems that facilitate and encourage decision makers to think visually. As technical barriers erode, computational and graphical processing capabilities will provide analysts and decision makers with new opportunities to develop understanding, enhance knowledge creation and ultimately provide decision support through more innovative, efficient and effective systems.

\section{References}

1 Hernandez, T. and Biasiotto, M. (2001) 'The status of corporate planning in Canada', CSCA Research Report, 2001-03, CSCA, Ryerson University, Toronto.

2 Andrienko, N., Andrienko, G. and Gatalsky, P. (2003) 'Exploratory spatio-temporal visualization: An analytical review', Journal of Visual Languages and Computing, Vol. 14, pp. 503-541.

3 Hernandez, T., Cornelilius, S. and Bennison, D. 
(1995) 'Finding spatial data: An evaluation of data sources for retail location analysis', Journal of Targeting, Measurement and Analysis for Marketing, Vol. 4, No. 1, pp. 11-24.

4 MacEachren, A. M. and Kraak, M. J. (2001) 'Research challenges in geovisualization', Cartography and Geographic Information Science, Vol. 28, No. 1, p. 3.

5 Brown, J., Earnshaw, R., Jern, M. and Vince, J. (1995) 'Visualization: Using computer graphics to explore data and present information', John Wiley \& Sons, New York.

6 Hearnshaw, H. and Unwin, D. (1994) 'Visualization in geographical information systems', The Association for Geographic Information, Sussex, UK.

7 Lloyd, R. (1997) 'Spatial cognition, geographic environments', Kluwer Academic Publishers, Dordrecht.

8 MacEachren, A. M. (1995) 'How maps work: Representation, visualization, and design', The Guildford Press, New York.

9 Slocum, T. A. (1999) 'Thematic cartography and visualization', Prentice Hall, Upper Saddle River, New Jersey.

10 Slocum, T. A., Blok, C., Jiang, B., Koussoulakou, A., Montello, D. R., Fuhrmann, S. and Hedley, N. R. (2001) 'Cognitive and usability issues in geovisualization', Cartography and Geographic Information Science, Vol. 28, No. 1, pp. 61-75.

11 MacEachren, A. M., Gahegan, M., Pike, W., Brewer, I., Cai, G. and Hardisty, F. (2003) 'Geovisualization for knowledge construction and decision-support', Computers and Applications, Vol. 24, pp. 13-17.

12 MacEachren, A. M. (1994) 'Visualization in modern cartography: Setting the agenda', in MacEachren, A. M. and Taylor, D. R. F. (eds) 'Visualization in modern cartography', Pergamon, Oxford, UK.

13 Bertin, J. (1981) 'Graphics and graphic information processing', Walter de Gruyter, Berlin.

14 Tufte, E. R. (1997) 'Visual explanations: Images and quantities, evidence and narrative', Graphics Press, Cheshire, Connecticut.

15 Tufte, E. R. (1990) 'Envisioning information', Graphics Press, Cheshire, Connecticut.

16 Slocum, T. A. and Egbert, S. L. (1993) 'Knowledge acquisition from choropleth maps', Cartography and Geographic Information Systems, Vol. 20, No. 2, pp. 83-95.

17 Haeberling, C. (1999) 'Symbolization in topographic 3D maps: Conceptual aspects for user-oriented design', in Proceedings of the 19th International Cartographic Conference, Ottawa, Canada, pp. 62-69.

18 Kraak, M. J. (1994) 'Interactive modelling environment for three-dimensional maps: Functionality and interface issues', in MacEachren, A. M. and Taylor, D. R. F. (eds) 'Visualization in modern cartography', Pergamon, Oxford.

19 Ledbetter, M. (1999) '3-D Visualization helps solve real-world problems', GEOWorld, Vol. 12, No. 9, pp. 52-54, 56.
20 Andrienko, G. L. and Andrienko, N. V. (1999) 'Interactive maps for visual data exploration', International Journal of Geographical Information Science, Vol. 13, No. 4, pp. 355-374.

21 DiBiase, D., MacEachren, A. M., Krygier, J. B. and Reeves, C. (1992) 'Animation and the role of map design in scientific visualization', Cartography and Geographic Information Systems, Vol. 19, No. 4, pp. 201-214, 265-266.

22 Bishop, I. D., Ramasamy, S. M., Stephens, P. and Joyce, E. B. (1999) 'Visualization of 8000 years of geological history in Southern India', International Journal of Geographical Information Science, Vol. 13, No. 4, pp. 417-427.

23 Koussoulakou, A. and Stylianidis, E. (1999) 'The use of GIS for the visual exploration of archaeological spatio-temporal data', Cartography and Geographic Information Science, Vol. 26, No. 2, pp. 152-160.

24 Dorling, D. (1992) 'Stretching space and splicing time: From cartographic animation to interactive visualization', Cartography and Geographic Information Systems, Vol. 19, No. 4, pp. 215-227, 267-270.

25 Acevedo, W. and Masuoka, P. (1997) 'Time-series animation techniques for visualizing urban growth', Computers \& Geosciences, Vol. 23, No. 4, pp. 423-435.

26 Blok, C., Köbben, B., Cheng, T. and Kuterema, A. A. (1999) 'Visualization of relationships between spatial patterns in time by cartographic animation', Cartography and Geographic Information Science, Vol. 26, No. 2, pp. 139-151.

27 Slocum (1999) op. cit.

28 Morrison, J. B., Tversky, B. and Betrancourt, M. (2000) 'Animation: Does it facilitate learning?', in AAAI Spring Symposium on Smart Graphics, Stanford, California, pp. 53-59.

29 Openshaw, S., Waugh, D. and Cross, A. (1993) 'Some ideas about the use of map animation as a spatial analysis tool', in Hearnshaw. H. M. and Unwin, D. J. (eds) 'Visualization in geographical information', John Wiley \& Sons, Chichester.

30 Robertson, G. G., Card, S. K. and Mackinlay, J. D. (1999) 'Information visualization using 3D interactive animation', in Card, S. K., Mackinlay, J. D. and Shneiderman, B. (eds) 'Readings in information visualization: Using vision to think', Morgan Kaufmann, San Francisco, California.

31 Slocum, T. A., Robeson, S. H. and Egbert, S. L. (1990) 'Traditional versus sequenced choropleth maps: An experimental investigation', Cartographica, Vol. 27, No. 1, pp. 67-88.

32 Slocum, T. A., Sluter, R. S., Kessler, F. C. and Yoder, S. C. (2000) 'A preliminary evaluation of MapTime', in Proceedings of Association of American Geographers Annual Meeting, Pittsburgh, Pennyslvania.

33 O'Rourke, J. (2001) 'Computational geometry in C', 2nd edn, Cambridge University Press, Cambridge, UK.

34 Buziek, G. (2000) 'Legend designs for noninteractive cartographic animations', Computers \& Geosciences, Vol. 26, pp. 21-28. 
35 Peterson, M. (1999) 'Active legends for interactive cartographic animation', International Journal of Geographical Information Science, Vol. 13, No. 4, pp. 375-383.

36 Kraak, M. J., Edsall, R. M. and MacEachren, A. M. (1997) 'Cartographic animation and legends for temporal maps', Proceeding of the 18th International Cartographic Association Conference, Stockholm, Sweden, ICA, pp. 253-262.

37 Centre for the Study of Commerical Activity (CSCA) (2004), Ryerson University, Toronto, http://www.businessgeomatics.com. 